\title{
Oscillation criteria of third-order nonlinear dynamic equations with nonpositive neutral coefficients on time scales
}

\section{Yang-Cong Qiu*}

\section{"Correspondence:} q840410@qq.com

School of Humanities and Social Science, Shunde Polytechnic, Foshan, Guangdong 528333, P.R. China

\begin{abstract}
In this paper, we establish oscillation criteria of third-order nonlinear dynamic equations with nonpositive neutral coefficients on time scales by a generalized Riccati transformation and employing functions in some function classes. Two examples are presented to show the significance of the results.
\end{abstract}

Keywords: third-order nonlinear dynamic equations; time scales; oscillation criteria; generalized Riccati transformation

\section{Introduction}

In this paper, we consider third-order nonlinear dynamic equations with nonpositive neutral coefficients of the form

$$
\left(r_{1}(t)\left(\left(r_{2}(t)\left(z^{\Delta}(t)\right)^{\gamma_{2}}\right)^{\Delta}\right)^{\gamma_{1}}\right)^{\Delta}+f(t, x(h(t)))=0,
$$

where $z(t)=x(t)-p(t) x(g(t))$, on a time scale $\mathbb{T}$ satisfying $\inf \mathbb{T}=t_{0}$ and $\sup \mathbb{T}=\infty$. Throughout this paper we assume that:

(C1) $r_{1}, r_{2} \in C_{\mathrm{rd}}(\mathbb{T},(0, \infty))$ such that

$$
\int_{t_{0}}^{\infty} \frac{1}{r_{1}^{1 / \gamma_{1}}(t)} \Delta t=\infty, \quad \int_{t_{0}}^{\infty} \frac{1}{r_{2}^{1 / \gamma_{2}}(t)} \Delta t=\infty ;
$$

(C2) $\gamma, \gamma_{1}, \gamma_{2}$ are all quotients of odd positive integers, and $\gamma=\gamma_{1} \cdot \gamma_{2}$;

(C3) $p \in C_{\mathrm{rd}}(\mathbb{T},[0, \infty))$ and there exists a constant $p_{0}$ with $0 \leq p_{0}<1$ such that

$$
\lim _{t \rightarrow \infty} p(t)=p_{0}
$$

(C4) $g \in C_{\mathrm{rd}}(\mathbb{T}, \mathbb{T}), g(t) \leq t, \lim _{t \rightarrow \infty} g(t)=\infty$, and there exists a sequence $\left\{c_{k}\right\}_{k \geq 0}$ such that $\lim _{k \rightarrow \infty} c_{k}=\infty$ and $g\left(c_{k+1}\right)=c_{k}$;

(C5) $h \in C_{\mathrm{rd}}(\mathbb{T}, \mathbb{T})$, and for any $t \in \mathbb{T}$,

$$
h(t) \geq \begin{cases}\sigma(t), & 0<\gamma<1, \\ t, & \gamma \geq 1\end{cases}
$$

(c) 2015 Qiu. This article is distributed under the terms of the Creative Commons Attribution 4.0 International License (http://creativecommons.org/licenses/by/4.0/), which permits unrestricted use, distribution, and reproduction in any medium, provided you give appropriate credit to the original author(s) and the source, provide a link to the Creative Commons license, and indicate if changes were made. 
(C6) $f \in C(\mathbb{T} \times \mathbb{R}, \mathbb{R})$ and there exists a function $q \in C_{\mathrm{rd}}(\mathbb{T},(0, \infty))$ such that $u f(t, u) \geq q(t) u^{\gamma+1}$

(C7) When $0<\gamma<1$, it always satisfies

$$
\int_{t_{0}}^{\infty} q(t) \Delta t<\infty
$$

Definition 1.1 A solution $x$ of (1) is said to have a generalized zero at $t^{*} \in \mathbb{T}$ if $x\left(t^{*}\right) x\left(\sigma\left(t^{*}\right)\right) \leq 0$, and it is said to be nonoscillatory on $\mathbb{T}$ if there exists $t_{0} \in \mathbb{T}$ such that $x(t) x(\sigma(t))>0$ for all $t>t_{0}$. Otherwise, it is oscillatory. Equation (1) is said to be oscillatory if all solutions of (1) are oscillatory.

In 1988, the theory of time scales was introduced by Hilger in his Ph.D. thesis [1] to unify continuous and discrete analysis; see also [2]. Since then, the theory had received a lot of attention. The details of time scales can be found in [3-6] and are omitted here.

There has been many achievements of the study of oscillation of nonlinear dynamic equations on time scales in the last few years; see [7-16] and the references therein. Hassan [8], Erbe et al. [7], and Zhang and Wang [16] gave some oscillation criteria successively for the third-order nonlinear delay dynamic equation

$$
\left(a(t)\left[\left(r(t) x^{\Delta}(t)\right)^{\Delta}\right]^{\gamma}\right)^{\Delta}+f(t, x(\tau(t)))=0 .
$$

Saker et al. [13] studied the oscillation of the second-order damped dynamic equation

$$
\left(a(t) x^{\Delta}(t)\right)^{\Delta}+p(t) x^{\Delta^{\sigma}}(t)+q(t)\left(f \circ x^{\sigma}\right)=0 .
$$

Qiu and Wang [10] considered second-order nonlinear dynamic equation

$$
\left(p(t) \psi(x(t)) k \circ x^{\Delta}(t)\right)^{\Delta}+f(t, x(\sigma(t)))=0 .
$$

Employing a generalized Riccati transformation

$$
u(t)=A(t) \frac{p(t) \psi(x(t)) k \circ x^{\Delta}(t)}{x(t)}+B(t)
$$

the authors established some Kamenev-type oscillation criteria. Şenel [14] investigated the oscillation of the second-order nonlinear dynamic equation of the form

$$
\left(r(t)\left(x^{\Delta}(t)\right)^{\gamma}\right)^{\Delta}+p(t)\left(x^{\Delta}(t)\right)^{\gamma}+f(t, x(g(t)))=0 .
$$

Qiu and Wang [11] corrected some mistakes in [14] and established correct oscillation criteria for (2). Yu and Wang [15] considered the third-order nonlinear dynamic equation

$$
\left(\frac{1}{a_{2}(t)}\left(\left(\frac{1}{a_{1}(t)}\left(x^{\Delta}(t)\right)^{\alpha_{1}}\right)^{\Delta}\right)^{\alpha_{2}}\right)^{\Delta}+q(t) f(x(t))=0
$$

under the condition $\alpha_{1} \alpha_{2}=1$, and they established some sufficient conditions which guarantee that every solution $x$ of (3) oscillates or converges to zero on a time scale $\mathbb{T}$. Li et al. 
[9] studied the second-order neutral delay differential equation

$$
\left(r(t)\left(z^{\prime}(t)\right)^{\alpha}\right)^{\prime}+q(t) f(x(\sigma(t)))=0, \quad t \geq t_{0}>0,
$$

where $z(t)=x(t)-p(t) x(\tau(t))$ and $\alpha>0$ is the ratio of two odd integers. Qiu [12] obtained some significant results for the existence of nonoscillatory solutions to the third-order nonlinear neutral dynamic equation of the form

$$
\left(r_{1}(t)\left(r_{2}(t)(x(t)+p(t) x(g(t)))^{\Delta}\right)^{\Delta}\right)^{\Delta}+f(t, x(h(t)))=0,
$$

where $\lim _{t \rightarrow \infty} p(t)=p_{0} \in(-1,1)$.

In this paper, motivated by $[9,10,12,14,15]$, we will establish oscillation criteria of (1), which are more general than (3), by a generalized Riccati transformation, and give two examples to show the significance of the results.

For the sake of simplicity, we denote $(a, b) \cap \mathbb{T}=(a, b)_{\mathbb{T}}$ throughout the paper, where $a, b \in \mathbb{R}$, and $[a, b]_{\mathbb{T}},[a, b)_{\mathbb{T}},(a, b]_{\mathbb{T}}$ are similar notations.

\section{Preliminary results}

To establish the oscillation criteria of (1), we give six lemmas in this section.

Lemma 2.1 Suppose that $x(t)$ is an eventually positive solution of (1), and there exists a constant $a \geq 0$ such that $\lim _{t \rightarrow \infty} z(t)=a$. Then we have

$$
\lim _{t \rightarrow \infty} x(t)=\frac{a}{1-p_{0}} .
$$

Proof Suppose that $x(t)$ is an eventually positive solution of (1). In view of (C3) and (C5), there exist $T \in\left[t_{0}, \infty\right)_{\mathbb{T}}$ and $p_{0}<p_{1}<1$ such that $x(t)>0, x(g(t))>0$, and $p(t) \leq p_{1}$ for $t \in[T, \infty)_{\mathbb{T}}$. We claim that $x(t)$ is bounded on $[T, \infty)_{\mathbb{T}}$. Assume not; then there exists $\left\{t_{n}\right\} \in[T, \infty)_{\mathbb{T}}$ with $t_{n} \rightarrow \infty$ as $n \rightarrow \infty$ such that

$$
x\left(t_{n}\right)=\max _{t \in\left[T, t_{n}\right]_{\mathbb{T}}} x(t) \text { and } \lim _{n \rightarrow \infty} x\left(t_{n}\right)=\infty .
$$

Noting that $g(t) \leq t$, we have

$$
z\left(t_{n}\right)=x\left(t_{n}\right)-p\left(t_{n}\right) x\left(g\left(t_{n}\right)\right) \geq\left(1-p_{1}\right) x\left(t_{n}\right) \rightarrow \infty
$$

as $n \rightarrow \infty$, which contradicts the fact that $\lim _{t \rightarrow \infty} z(t)=a$. Therefore, $x(t)$ is bounded. Then assume that

$$
\limsup _{t \rightarrow \infty} x(t)=\bar{x} \quad \text { and } \quad \liminf _{t \rightarrow \infty} x(t)=\underline{x} .
$$

Since $0 \leq p_{0}<1$, we have

$$
a \geq \bar{x}-p_{0} \bar{x} \text { and } a \leq \underline{x}-p_{0} \underline{x},
$$

which implies that $\bar{x} \leq \underline{x}$. So $\bar{x}=\underline{x}$, and we see that $\lim _{t \rightarrow \infty} x(t)$ exists and $\lim _{t \rightarrow \infty} x(t)=$ $a /\left(1-p_{0}\right)$. The proof is complete. 
Lemma 2.2 Assume that $x(t)$ is an eventually positive solution of $(1)$, then there exists a sufficiently large $T \in\left[t_{0}, \infty\right)_{\mathbb{T}}$ such that, for $t \in[T, \infty)_{\mathbb{T}}$, we have

$$
\left(r_{2}(t)\left(z^{\Delta}(t)\right)^{\gamma_{2}}\right)^{\Delta}>0
$$

and

$$
z^{\Delta}(t)>0 \quad \text { or } \quad z^{\Delta}(t)<0 .
$$

Proof Suppose that $x(t)$ is an eventually positive solution of (1). From (C3) and (C5), there exist $t_{1} \in\left[t_{0}, \infty\right)_{\mathbb{T}}$ and $p_{0}<p_{1}<1$ such that $x(t)>0, x(g(t))>0, x(h(t))>0$, and $p(t) \leq p_{1}$ for $t \in\left[t_{1}, \infty\right)_{\mathbb{T}}$. By $(1)$ and $(\mathrm{C} 6)$, it follows that, for $t \in\left[t_{1}, \infty\right)_{\mathbb{T}}$,

$$
\left(r_{1}(t)\left(\left(r_{2}(t)\left(z^{\Delta}(t)\right)^{\gamma_{2}}\right)^{\Delta}\right)^{\gamma_{1}}\right)^{\Delta}=-f(t, x(h(t)))<0 .
$$

Hence, $r_{1}(t)\left(\left(r_{2}(t)\left(z^{\Delta}(t)\right)^{\gamma_{2}}\right)^{\Delta}\right)^{\gamma_{1}}$ is strictly decreasing on $\left[t_{1}, \infty\right)_{\mathbb{T}}$. We claim that

$$
r_{1}(t)\left(\left(r_{2}(t)\left(z^{\Delta}(t)\right)^{\gamma_{2}}\right)^{\Delta}\right)^{\gamma_{1}}>0, \quad t \in\left[t_{1}, \infty\right)_{\mathbb{T}} .
$$

Assume not; then there exists $t_{2} \in\left[t_{1}, \infty\right)_{\mathbb{T}}$ such that

$$
r_{1}(t)\left(\left(r_{2}(t)\left(z^{\Delta}(t)\right)^{\gamma_{2}}\right)^{\Delta}\right)^{\gamma_{1}}<0
$$

for $t \in\left[t_{2}, \infty\right)_{\mathbb{T}}$. So there exists a constant $c<0$ and we have $t_{3} \in\left[t_{2}, \infty\right)_{\mathbb{T}}$ such that $r_{1}(t)\left(\left(r_{2}(t)\left(z^{\Delta}(t)\right)^{\gamma_{2}}\right)^{\Delta}\right)^{\gamma_{1}} \leq c$ for $t \in\left[t_{3}, \infty\right)_{\mathbb{T}}$, which means that

$$
\left(r_{2}(t)\left(z^{\Delta}(t)\right)^{\gamma_{2}}\right)^{\Delta} \leq\left(\frac{c}{r_{1}(t)}\right)^{1 / \gamma_{1}}, \quad t \in\left[t_{3}, \infty\right)_{\mathbb{T}} .
$$

Substituting $s$ for $t$, and integrating (6) from $t_{3}$ to $t \in\left[\sigma\left(t_{3}\right), \infty\right)_{\mathbb{T}}$, we obtain

$$
r_{2}(t)\left(z^{\Delta}(t)\right)^{\gamma_{2}} \leq r_{2}\left(t_{3}\right)\left(z^{\Delta}\left(t_{3}\right)\right)^{\gamma_{2}}+c^{1 / \gamma_{1}} \int_{t_{3}}^{t} \frac{\Delta s}{r_{1}^{1 / \gamma_{1}}(s)} .
$$

Letting $t \rightarrow \infty$, by $(\mathrm{C} 1)$ we have $r_{2}(t)\left(z^{\Delta}(t)\right)^{\gamma_{2}} \rightarrow-\infty$. Then there exists $t_{4} \in\left[t_{3}, \infty\right)_{\mathbb{T}}$ such that $r_{2}(t)\left(z^{\Delta}(t)\right)^{\gamma_{2}} \leq r_{2}\left(t_{4}\right)\left(z^{\Delta}\left(t_{4}\right)\right)^{\gamma_{2}}<0$ for $t \in\left[t_{4}, \infty\right)_{\mathbb{T}}$, which implies that

$$
z^{\Delta}(t) \leq r_{2}^{1 / \gamma_{2}}\left(t_{4}\right) z^{\Delta}\left(t_{4}\right) \cdot \frac{1}{r_{2}^{1 / \gamma_{2}}(t)}
$$

Substituting $s$ for $t$, and integrating (7) from $t_{4}$ to $t \in\left[\sigma\left(t_{4}\right), \infty\right)_{\mathbb{T}}$, we obtain

$$
z(t)-z\left(t_{4}\right) \leq r_{2}^{1 / \gamma_{2}}\left(t_{4}\right) z^{\Delta}\left(t_{4}\right) \int_{t_{4}}^{t} \frac{\Delta s}{r_{2}^{1 / \gamma_{2}}(s)} .
$$

Letting $t \rightarrow \infty$, by (C1) we have $z(t) \rightarrow-\infty$. Then there exists $t_{5} \in\left[t_{4}, \infty\right)_{\mathbb{T}}$ such that $z(t)<0$ or

$$
x(t)<p(t) x(g(t)) \leq p_{1} x(g(t)), \quad t \in\left[t_{5}, \infty\right)_{\mathbb{T}} .
$$


By (C4), we can choose some positive integer $k_{0}$ such that $c_{k} \in\left[t_{5}, \infty\right)_{\mathbb{T}}$ for all $k \geq k_{0}$. Then for any $k \geq k_{0}+1$, we have

$$
\begin{aligned}
x\left(c_{k}\right) & <p_{1} x\left(g\left(c_{k}\right)\right)=p_{1} x\left(c_{k-1}\right)<p_{1}^{2} x\left(g\left(c_{k-1}\right)\right)=p_{1}^{2} x\left(c_{k-2}\right)<\cdots \\
& <p_{1}^{k-k_{0}} x\left(g\left(c_{k_{0}+1}\right)\right)=p_{1}^{k-k_{0}} x\left(c_{k_{0}}\right) .
\end{aligned}
$$

The inequality above implies that $\lim _{k \rightarrow \infty} x\left(c_{k}\right)=0$. It follows that

$$
\lim _{k \rightarrow \infty} z\left(c_{k}\right)=0
$$

and this contradicts $\lim _{t \rightarrow \infty} z(t)=-\infty$. So (5) holds, which implies that

$$
\left(r_{2}(t)\left(z^{\Delta}(t)\right)^{\gamma_{2}}\right)^{\Delta}>0, \quad t \in\left[t_{1}, \infty\right)_{\mathbb{T}} .
$$

Therefore, $r_{2}(t)\left(z^{\Delta}(t)\right)^{\gamma_{2}}$ is strictly increasing on $\left[t_{1}, \infty\right)_{\mathbb{T}}$. It follows that $r_{2}(t)\left(z^{\Delta}(t)\right)^{\gamma_{2}}$ is eventually positive or $r_{2}(t)\left(z^{\Delta}(t)\right)^{\gamma_{2}}<0$ on $\left[t_{1}, \infty\right)_{\mathbb{T}}$. Lemma 2.2 is proved.

Lemma 2.3 Assume that $x(t)$ is an eventually positive solution of $(1)$, then $z(t)$ is eventually positive or $\lim _{t \rightarrow \infty} x(t)=0$.

Proof Suppose that $x(t)$ is an eventually positive solution of (1), by Lemma 2.2 there exists $t_{1} \in\left[t_{0}, \infty\right)_{\mathbb{T}}$ such that $z^{\Delta}(t)>0$ or $z^{\Delta}(t)<0, t \in\left[t_{1}, \infty\right)_{\mathbb{T}}$.

(i) $z^{\Delta}(t)>0, t \in\left[t_{1}, \infty\right)_{\mathbb{T}}$. Then it follows that $z(t)$ is eventually positive or eventually negative. If $z(t)$ is eventually positive, the lemma is proved. If $z(t)$ is eventually negative, we see that $\lim _{t \rightarrow \infty} z(t)$ exists. Assume that $\lim _{t \rightarrow \infty} z(t)<0$. Similarly as in the proof of Lemma 2.2, we will have the contradiction. Hence, $\lim _{t \rightarrow \infty} z(t)=0$. Then it follows that $\lim _{t \rightarrow \infty} x(t)=0$ by Lemma 2.1 .

(ii) $z^{\Delta}(t)<0, t \in\left[t_{1}, \infty\right)_{\mathbb{T}}$. Similarly, we see that $z(t)$ is eventually positive or eventually negative. Assume that $z(t)$ is eventually negative, there exists a constant $c<0$ and we have $t_{2} \in\left[t_{1}, \infty\right)_{\mathbb{T}}$ such that $z(t)<c, t \in\left[t_{2}, \infty\right)_{\mathbb{T}}$. It will cause a similar contradiction as in the proof of Lemma 2.2. Hence, $z(t)$ is eventually positive and the lemma is proved.

The proof is complete.

Lemma 2.4 For $0<\gamma<1$, assume that $x(t)$ is an eventually positive solution of (1), and $z(t), z^{\Delta}(t)$ are both eventually positive. Then there exists $t_{1} \in\left[t_{0}, \infty\right)_{\mathbb{T}}$ such that

$$
\left(\frac{z^{\Delta}(t)}{z^{\sigma}(t)}\right)^{1-\gamma} \geq \alpha(t)=\left(\frac{\delta(t)}{r_{2}(t)}\right)^{(1-\gamma) / \gamma_{2}}\left(\int_{t}^{\infty} q(s) \Delta s\right)^{(1-\gamma) / \gamma}, \quad t \in\left[t_{1}, \infty\right)_{\mathbb{T}}
$$

where

$$
\delta(t)=\int_{t_{1}}^{t} \frac{\Delta s}{r_{1}^{1 / \gamma_{1}}(s)} .
$$

Proof Suppose that $x(t)$ is an eventually positive solution of (1), and $z(t), z^{\Delta}(t)$ are both eventually positive, then there exists $t_{1} \in\left[t_{0}, \infty\right)_{\mathbb{T}}$ such that $x(t)>0, x(g(t))>0, x(h(t))>0$, 
$z(t)>0$, and $z^{\Delta}(t)>0$ for $t \in\left[t_{1}, \infty\right)_{\mathbb{T}}$. By Lemma 2.2 we have

$$
\left(r_{2}(t)\left(z^{\Delta}(t)\right)^{\gamma_{2}}\right)^{\Delta}>0, \quad t \in\left[t_{1}, \infty\right)_{\mathbb{T}} .
$$

By $z^{\Delta}(t)>0$ and $z(t)=x(t)-p(t) x(g(t)) \leq x(t)$, it follows that, for $t \in\left[t_{1}, \infty\right)_{\mathbb{T}}$,

$$
\begin{aligned}
& \left(r_{1}(t)\left(\left(r_{2}(t)\left(z^{\Delta}(t)\right)^{\gamma_{2}}\right)^{\Delta}\right)^{\gamma_{1}}\right)^{\Delta} \\
& \quad=-f(t, x(h(t))) \leq-q(t) x^{\gamma}(h(t)) \leq-q(t) z^{\gamma}(h(t)) \leq-q(t) z^{\gamma}(\sigma(t))<0 .
\end{aligned}
$$

Substituting $s$ for $t$, and integrating (8) from $t \in\left[t_{1}, \infty\right)_{\mathbb{T}}$ to $\infty$, we obtain

$$
r_{1}(t)\left(\left(r_{2}(t)\left(z^{\Delta}(t)\right)^{\gamma_{2}}\right)^{\Delta}\right)^{\gamma_{1}} \geq \int_{t}^{\infty} q(s) z^{\gamma}(\sigma(s)) \Delta s \geq z^{\gamma}(\sigma(t)) \int_{t}^{\infty} q(s) \Delta s .
$$

As $r_{1}(t)\left(\left(r_{2}(t)\left(z^{\Delta}(t)\right)^{\gamma_{2}}\right)^{\Delta}\right)^{\gamma_{1}}$ is strictly decreasing on $\left[t_{1}, \infty\right)_{\mathbb{T}}$, we have, for $t \in\left[\sigma\left(t_{1}\right), \infty\right)_{\mathbb{T}}$,

$$
\begin{aligned}
r_{2}(t)\left(z^{\Delta}(t)\right)^{\gamma_{2}} & =r_{2}\left(t_{1}\right)\left(z^{\Delta}\left(t_{1}\right)\right)^{\gamma_{2}}+\int_{t_{1}}^{t} \frac{r_{1}^{1 / \gamma_{1}}(s)\left(r_{2}(s)\left(z^{\Delta}(s)\right)^{\gamma_{2}}\right)^{\Delta}}{r_{1}^{1 / \gamma_{1}}(s)} \Delta s \\
& \geq r_{1}^{1 / \gamma_{1}}(t)\left(r_{2}(t)\left(z^{\Delta}(t)\right)^{\gamma_{2}}\right)^{\Delta} \int_{t_{1}}^{t} \frac{1}{r_{1}^{1 / \gamma_{1}}(s)} \Delta s \\
& \geq \delta(t)\left(z^{\gamma}(\sigma(t)) \int_{t}^{\infty} q(s) \Delta s\right)^{1 / \gamma_{1}}=\delta(t) z^{\gamma_{2}}(\sigma(t))\left(\int_{t}^{\infty} q(s) \Delta s\right)^{1 / \gamma_{1}} .
\end{aligned}
$$

Hence, when $0<\gamma<1$, we have

$$
\frac{z^{\Delta}(t)}{z^{\sigma}(t)} \geq\left(\frac{\delta(t)}{r_{2}(t)}\right)^{1 / \gamma_{2}}\left(\int_{t}^{\infty} q(s) \Delta s\right)^{1 / \gamma}, \quad t \in\left[t_{1}, \infty\right)_{\mathbb{T}}
$$

which implies that

$$
\left(\frac{z^{\Delta}(t)}{z^{\sigma}(t)}\right)^{1-\gamma} \geq \alpha(t), \quad t \in\left[t_{1}, \infty\right)_{\mathbb{T}} .
$$

Lemma 2.4 is proved.

Lemma 2.5 For $\gamma \geq 1$, assume that $x(t)$ is an eventually positive solution of $(1)$, and $z^{\Delta}(t)$ is eventually negative. If it satisfies

$$
\int_{t_{0}}^{\infty} q(t) \Delta t=\infty
$$

then $\lim _{t \rightarrow \infty} x(t)=0$.

Proof Suppose that $x(t)$ is an eventually positive solution of $(1)$ and $z^{\Delta}(t)$ is eventually negative. By the proof of Lemma 2.3, we see that $z(t)$ is eventually positive. Then there exists $t_{1} \in\left[t_{0}, \infty\right)_{\mathbb{T}}$ such that $x(t)>0, x(g(t))>0, x(h(t))>0, z(t)>0$, and $z^{\Delta}(t)<0$ for $t \in\left[t_{1}, \infty\right)_{\mathbb{T}}$. By Lemma 2.2 we have

$$
\left(r_{2}(t)\left(z^{\Delta}(t)\right)^{\gamma_{2}}\right)^{\Delta}>0, \quad t \in\left[t_{1}, \infty\right)_{\mathbb{T}} .
$$


By $z^{\Delta}(t)<0$, we claim that there exists $b \geq 0$ such that $\lim _{t \rightarrow \infty} z(t)=b$. Assume not; then there exists $t_{2} \in\left[t_{1}, \infty\right)_{\mathbb{T}}$ such that $z(t)<0$ for $t \in\left[t_{2}, \infty\right)_{\mathbb{T}}$. It will cause a similar contradiction as in the proof of Lemma 2.2. Then assuming $b>0$, by $(8)$ and $z(\sigma(t)), z(g(t))>b$, we obtain

$$
\left(r_{1}(t)\left(\left(r_{2}(t)\left(z^{\Delta}(t)\right)^{\gamma_{2}}\right)^{\Delta}\right)^{\gamma_{1}}\right)^{\Delta} \leq-q(t) z^{\gamma}(\sigma(t))<-b^{\gamma} q(t)
$$

Letting $v(t)=r_{1}(t)\left(\left(r_{2}(t)\left(z^{\Delta}(t)\right)^{\gamma_{2}}\right)^{\Delta}\right)^{\gamma_{1}}, t \in\left[t_{1}, \infty\right)_{\mathbb{T}}$, we have $v(t)>0$, and

$$
v^{\Delta}(t)<-b^{\gamma} q(t), \quad t \in\left[t_{1}, \infty\right)_{\mathbb{T}}
$$

Substituting $s$ for $t$, and integrating (11) from $t_{1}$ to $t \in\left[\sigma\left(t_{1}\right), \infty\right)_{\mathbb{T}}$, we obtain

$$
v(t)<v\left(t_{1}\right)-b^{\gamma} \int_{t_{1}}^{t} q(s) \Delta s
$$

By (9), there exists a sufficiently large $t_{2} \in\left[t_{1}, \infty\right)_{\mathbb{T}}$ such that $v(t)<0, t \in\left[t_{2}, \infty\right)_{\mathbb{T}}$, which contradicts $v(t)>0$. So $b=0$, and Lemma 2.5 is proved.

Lemma 2.6 Assume that $x(t)$ is an eventually positive solution of (1), and there exists $t_{1} \in$ $\left[t_{0}, \infty\right)_{\mathbb{T}}$ such that $x(t)>0, x(g(t))>0, x(h(t))>0, z(t)>0$, and $z^{\Delta}(t)>0$ for $t \in\left[t_{1}, \infty\right)_{\mathbb{T}}$. For $t \in\left[t_{1}, \infty\right)_{\mathbb{T}}$, define

$$
u(t)=A(t) \frac{r_{1}(t)\left(\left(r_{2}(t)\left(z^{\Delta}(t)\right)^{\gamma_{2}}\right)^{\Delta}\right)^{\gamma_{1}}}{z^{\gamma}(t)}+B(t)
$$

where $A \in C_{\mathrm{rd}}^{1}(\mathbb{T},(0, \infty)), B \in C_{\mathrm{rd}}^{1}(\mathbb{T}, \mathbb{R})$. Then $u(t)$ satisfies

$$
u^{\Delta}(t)+A(t) q(t)-B^{\Delta}(t)-\Phi_{0}(t) \leq 0
$$

where

$$
\Phi_{0}(t)= \begin{cases}A^{\Delta}(t)\left(\frac{u(t)-B(t)}{A(t)}\right)^{\sigma}-\gamma A(t) \alpha(t)\left(\frac{\delta(t)}{r_{2}(t)}\right)^{\gamma_{1}}\left[\left(\frac{u(t)-B(t)}{A(t)}\right)^{\sigma}\right]^{2}, & 0<\gamma<1, \\ A^{\Delta}(t)\left(\frac{u(t)-B(t)}{A(t)}\right)^{\sigma}-\gamma A(t)\left(\frac{\delta(t)}{r_{2}(t)}\right)^{1 / \gamma_{2}}\left[\left(\frac{u(t)-B(t)}{A(t)}\right)^{\sigma}\right]^{(1+\gamma) / \gamma}, & \gamma \geq 1 .\end{cases}
$$

Proof Since $x(t)$ is an eventually positive solution of $(1)$, and there exists $t_{1} \in\left[t_{0}, \infty\right)_{\mathbb{T}}$ such that $x(t)>0, x(g(t))>0, x(h(t))>0, z(t)>0$, and $z^{\Delta}(t)>0$ for $t \in\left[t_{1}, \infty\right)_{\mathbb{T}}$, Lemmas 2.2 and 2.4 hold. Let $u(t)$ be defined by (12). Then, differentiating (12) and using (1), it follows that

$$
\begin{aligned}
u^{\Delta}(t)= & \frac{A(t)}{z^{\gamma}(t)}\left(r_{1}(t)\left(\left(r_{2}(t)\left(z^{\Delta}(t)\right)^{\gamma_{2}}\right)^{\Delta}\right)^{\gamma_{1}}\right)^{\Delta} \\
& +\left(\frac{A(t)}{z^{\gamma}(t)}\right)^{\Delta}\left(r_{1}(t)\left(\left(r_{2}(t)\left(z^{\Delta}(t)\right)^{\gamma_{2}}\right)^{\Delta}\right)^{\gamma_{1}}\right)^{\sigma}+B^{\Delta}(t) \\
= & -\frac{A(t)}{z^{\gamma}(t)} \cdot f(t, x(h(t)))+B^{\Delta}(t) \\
& +\frac{A^{\Delta}(t) z^{\gamma}(t)-A(t)\left(z^{\gamma}(t)\right)^{\Delta}}{z^{\gamma}(t) z^{\gamma}(\sigma(t))}\left(r_{1}(t)\left(\left(r_{2}(t)\left(z^{\Delta}(t)\right)^{\gamma_{2}}\right)^{\Delta}\right)^{\gamma_{1}}\right)^{\sigma} .
\end{aligned}
$$


Using the fact that

$$
f(t, x(h(t))) \geq q(t) x^{\gamma}(h(t)) \geq q(t) z^{\gamma}(h(t)) \geq q(t) z^{\gamma}(t)
$$

we obtain

$$
\begin{aligned}
u^{\Delta}(t) \leq & -A(t) q(t)+B^{\Delta}(t)+A^{\Delta}(t)\left(\frac{u(t)-B(t)}{A(t)}\right)^{\sigma} \\
& -A(t) \frac{\left(z^{\gamma}(t)\right)^{\Delta}}{z^{\gamma}(t)}\left(\frac{u(t)-B(t)}{A(t)}\right)^{\sigma} .
\end{aligned}
$$

When $0<\gamma<1$, using the Pötzsche chain rule (see [5]), we have

$$
\left(z^{\gamma}(t)\right)^{\Delta}=\gamma \int_{0}^{1}\left(z(t)+h \mu(t) z^{\Delta}(t)\right)^{\gamma-1} d h \cdot z^{\Delta}(t) \geq \gamma\left(z^{\sigma}(t)\right)^{\gamma-1} z^{\Delta}(t),
$$

and it follows that

$$
\frac{\left(z^{\gamma}(t)\right)^{\Delta}}{z^{\gamma}(t)} \geq \frac{\gamma\left(z^{\sigma}(t)\right)^{\gamma-1} z^{\Delta}(t)}{z^{\gamma}(t)}=\gamma \frac{z^{\Delta}(t)}{z^{\sigma}(t)}\left(\frac{z^{\sigma}(t)}{z(t)}\right)^{\gamma} .
$$

By Lemmas 2.2 and 2.4 , for $t \in\left[t_{1}, \infty\right)_{\mathbb{T}}$, we obtain

$$
\begin{aligned}
\frac{z^{\Delta}(t)}{z^{\sigma}(t)} & =\frac{1}{r_{2}^{\gamma_{1}}(t)} \frac{r_{2}^{\gamma_{1}}(t)\left(z^{\Delta}(t)\right)^{\gamma}}{\left(z^{\sigma}(t)\right)^{\gamma}}\left(\frac{z^{\Delta}(t)}{z^{\sigma}(t)}\right)^{1-\gamma} \\
& \geq \alpha(t)\left(\frac{\delta(t)}{r_{2}(t)}\right)^{\gamma_{1}} \frac{r_{1}(t)\left(\left(r_{2}(t)\left(z^{\Delta}(t)\right)^{\gamma_{2}}\right)^{\Delta}\right)^{\gamma_{1}}}{\left(z^{\gamma}(t)\right)^{\sigma}} \\
& \geq \alpha(t)\left(\frac{\delta(t)}{r_{2}(t)}\right)^{\gamma_{1}} \frac{\left(r_{1}(t)\left(\left(r_{2}(t)\left(z^{\Delta}(t)\right)^{\gamma_{2}}\right)^{\Delta}\right)^{\gamma_{1}}\right)^{\sigma}}{\left(z^{\gamma}(t)\right)^{\sigma}} \\
& =\alpha(t)\left(\frac{\delta(t)}{r_{2}(t)}\right)^{\gamma_{1}}\left(\frac{u(t)-B(t)}{A(t)}\right)^{\sigma}
\end{aligned}
$$

and

$$
\frac{z^{\sigma}(t)}{z(t)} \geq 1
$$

So (14) becomes

$$
\begin{aligned}
u^{\Delta}(t) \leq & -A(t) q(t)+B^{\Delta}(t)+A^{\Delta}(t)\left(\frac{u(t)-B(t)}{A(t)}\right)^{\sigma} \\
& -\gamma A(t) \alpha(t)\left(\frac{\delta(t)}{r_{2}(t)}\right)^{\gamma_{1}}\left[\left(\frac{u(t)-B(t)}{A(t)}\right)^{\sigma}\right]^{2} .
\end{aligned}
$$

When $\gamma \geq 1$, we have

$$
\left(z^{\gamma}(t)\right)^{\Delta}=\gamma \int_{0}^{1}\left(z(t)+h \mu(t) z^{\Delta}(t)\right)^{\gamma-1} d h \cdot z^{\Delta}(t) \geq \gamma z^{\gamma-1}(t) z^{\Delta}(t),
$$


and it follows that

$$
\frac{\left(z^{\gamma}(t)\right)^{\Delta}}{z^{\gamma}(t)} \geq \frac{\gamma z^{\gamma-1}(t) z^{\Delta}(t)}{z^{\gamma}(t)}=\frac{\gamma z^{\Delta}(t)}{z(t)}
$$

By Lemmas 2.2 and 2.4 , for $t \in\left[t_{1}, \infty\right)_{\mathbb{T}}$, we obtain

$$
\begin{aligned}
\left(\frac{z^{\Delta}(t)}{z(t)}\right)^{\gamma} & =\frac{1}{r_{2}^{\gamma_{1}}(t)} \frac{r_{2}^{\gamma_{1}}(t)\left(z^{\Delta}(t)\right)^{\gamma}}{z^{\gamma}(t)} \\
& \geq\left(\frac{\delta(t)}{r_{2}(t)}\right)^{\gamma_{1}} \frac{r_{1}(t)\left(\left(r_{2}(t)\left(z^{\Delta}(t)\right)^{\gamma_{2}}\right)^{\Delta}\right)^{\gamma_{1}}}{z^{\gamma}(t)} \\
& \geq\left(\frac{\delta(t)}{r_{2}(t)}\right)^{\gamma_{1}} \frac{\left(r_{1}(t)\left(\left(r_{2}(t)\left(z^{\Delta}(t)\right)^{\gamma_{2}}\right)^{\Delta}\right)^{\gamma_{1}}\right)^{\sigma}}{\left(z^{\gamma}(t)\right)^{\sigma}} \\
& =\left(\frac{\delta(t)}{r_{2}(t)}\right)^{\gamma_{1}}\left(\frac{u(t)-B(t)}{A(t)}\right)^{\sigma},
\end{aligned}
$$

which implies that

$$
\frac{z^{\Delta}(t)}{z(t)} \geq\left(\frac{\delta(t)}{r_{2}(t)}\right)^{1 / \gamma_{2}}\left[\left(\frac{u(t)-B(t)}{A(t)}\right)^{\sigma}\right]^{1 / \gamma}
$$

So (14) becomes

$$
\begin{aligned}
u^{\Delta}(t) \leq & -A(t) q(t)+B^{\Delta}(t)+A^{\Delta}(t)\left(\frac{u(t)-B(t)}{A(t)}\right)^{\sigma} \\
& -\gamma A(t)\left(\frac{\delta(t)}{r_{2}(t)}\right)^{1 / \gamma_{2}}\left[\left(\frac{u(t)-B(t)}{A(t)}\right)^{\sigma}\right]^{(1+\gamma) / \gamma} .
\end{aligned}
$$

By (15) and (16), (13) holds. Lemma 2.6 is proved.

\section{Main results}

In this section, we establish oscillation criteria of (1) by a generalized Riccati transformation. Firstly, we give some definitions as follows.

Let $D_{0}=\{s \in \mathbb{T}: s \geq 0\}$ and $D=\left\{(t, s) \in \mathbb{T}^{2}: t \geq s \geq 0\right\}$. For any function $f(t, s): \mathbb{T}^{2} \rightarrow \mathbb{R}$, denote by $f_{2}^{\Delta}$ the partial derivative of $f$ with respect to $s$. Define

$$
\begin{aligned}
& (\mathscr{A}, \mathscr{B})=\left\{(A, B): A(s) \in C_{\mathrm{rd}}^{1}\left(D_{0},(0, \infty)\right), B(s) \in C_{\mathrm{rd}}^{1}\left(D_{0}, \mathbb{R}\right), s \in D_{0}\right\} \\
& \mathscr{H}=\left\{H(t, s) \in C^{1}(D,[0, \infty)): H(t, t)=0, H(t, s)>0, H_{2}^{\Delta}(t, s) \leq 0, t>s \geq 0\right\} .
\end{aligned}
$$

These function classes will be used in the sequel. Now, we give our first theorem.

Theorem 3.1 Assume that there exist $(A, B) \in(\mathscr{A}, \mathscr{B})$ and $H \in \mathscr{H}$ such that, for any $t_{1} \in$ $\left[t_{0}, \infty\right)_{\mathbb{T}}$,

$$
\limsup _{t \rightarrow \infty} \frac{1}{H\left(t, t_{1}\right)} \int_{t_{1}}^{t}\left[H(t, s)\left(A(s) q(s)-B^{\Delta}(s)\right)-H_{2}^{\Delta}(t, s) B^{\sigma}(s)-\Phi_{1}(s)\right] \Delta s=\infty,
$$


where

$$
\Phi_{1}(s)= \begin{cases}\left(\frac{r_{2}(s)}{\delta(s)}\right)^{\gamma_{1}} \frac{\left(H_{2}^{\Delta}(t, s) A^{\sigma}(s)+H(t, s) A^{\Delta}(s)\right)^{2}}{4 \gamma H(t, s) A(s) \alpha(s)}, & 0<\gamma<1, \\ \left(\frac{r_{2}(s)}{\delta(s)}\right)^{\gamma_{1}} \frac{1}{(H(t, s) A(s))^{\gamma}}\left(\frac{H_{2}^{\Delta}(t, s) A^{\sigma}(s)+H(t, s) A^{\Delta}(s)}{1+\gamma}\right)^{1+\gamma}, & \gamma \geq 1 .\end{cases}
$$

Then (1) is oscillatory or $\lim _{t \rightarrow \infty} x(t)$ exists.

Proof Assume that (1) is not oscillatory. Without loss of generality, we may suppose that $x(t)$ is an eventually positive solution of (1). Then by Lemma 2.3 , we have $z(t)$ is eventually positive or $\lim _{t \rightarrow \infty} x(t)=0$.

If $\lim _{t \rightarrow \infty} x(t)=0$, the theorem is proved. While $z(t)$ is eventually positive, it follows that there exists $T \in\left[t_{0}, \infty\right)_{\mathbb{T}}$ such that $z(t)>0$ for $t \in[T, \infty)_{\mathbb{T}}$. By Lemma 2.2, there exists $t_{1} \in[T, \infty)_{\mathbb{T}}$ such that either $z^{\Delta}(t)>0$ or $z^{\Delta}(t)<0$ holds for $t \in\left[t_{1}, \infty\right)_{\mathbb{T}}$. Assume that $z^{\Delta}(t)>0, t \in\left[t_{1}, \infty\right)_{\mathbb{T}}$. Let $u(t)$ be defined by (12). Then by Lemma 2.6, (13) holds.

Multiplying (13), where $t$ is replaced by $s$, by $H$, and integrating it with respect to $s$ from $t_{1}$ to $t$ with $t \in\left[\sigma\left(t_{1}\right), \infty\right)_{\mathbb{T}}$, we obtain

$$
\begin{aligned}
& \int_{t_{1}}^{t} H(t, s)\left(A(s) q(s)-B^{\Delta}(s)\right) \Delta s \\
& \quad \leq-\int_{t_{1}}^{t} H(t, s) u^{\Delta}(s) \Delta s+\int_{t_{1}}^{t} H(t, s) \Phi_{0}(s) \Delta s .
\end{aligned}
$$

Noting that $H(t, t)=0$, by the integration by parts formula we have

$$
\begin{aligned}
\int_{t_{1}}^{t} H(t, s)\left(A(s) q(s)-B^{\Delta}(s)\right) \Delta s \\
\leq H\left(t, t_{1}\right) u\left(t_{1}\right)+\int_{t_{1}}^{t}\left(H_{2}^{\Delta}(t, s) u^{\sigma}(s)+H(t, s) \Phi_{0}(s)\right) \Delta s \\
=H\left(t, t_{1}\right) u\left(t_{1}\right)+\int_{t_{1}}^{t} H_{2}^{\Delta}(t, s) B^{\sigma}(s) \Delta s \\
\quad+\int_{t_{1}}^{t}\left(H_{2}^{\Delta}(t, s) A^{\sigma}(s)\left(\frac{u(s)-B(s)}{A(s)}\right)^{\sigma}+H(t, s) \Phi_{0}(s)\right) \Delta s .
\end{aligned}
$$

When $0<\gamma<1$, we have

$$
\begin{gathered}
H_{2}^{\Delta}(t, s) A^{\sigma}(s)\left(\frac{u(s)-B(s)}{A(s)}\right)^{\sigma}+H(t, s) \Phi_{0}(s) \\
=\left(H_{2}^{\Delta}(t, s) A^{\sigma}(s)+H(t, s) A^{\Delta}(s)\right)\left(\frac{u(s)-B(s)}{A(s)}\right)^{\sigma} \\
\quad-\gamma H(t, s) A(s) \alpha(s)\left(\frac{\delta(s)}{r_{2}(s)}\right)^{\gamma_{1}}\left[\left(\frac{u(s)-B(s)}{A(s)}\right)^{\sigma}\right]^{2} \\
=\left(\frac{r_{2}(s)}{\delta(s)}\right)^{\gamma_{1}} \frac{\left(H_{2}^{\Delta}(t, s) A^{\sigma}(s)+H(t, s) A^{\Delta}(s)\right)^{2}}{4 \gamma H(t, s) A(s) \alpha(s)} \\
\quad-\gamma H(t, s) A(s) \alpha(s)\left(\frac{\delta(s)}{r_{2}(s)}\right)^{\gamma_{1}}\left[\left(\frac{u(s)-B(s)}{A(s)}\right)^{\sigma}\right.
\end{gathered}
$$




$$
\begin{aligned}
& \left.-\left(\frac{r_{2}(s)}{\delta(s)}\right)^{\gamma_{1}} \frac{H_{2}^{\Delta}(t, s) A^{\sigma}(s)+H(t, s) A^{\Delta}(s)}{2 \gamma H(t, s) A(s) \alpha(s)}\right]^{2} \\
\leq & \left(\frac{r_{2}(s)}{\delta(s)}\right)^{\gamma_{1}} \frac{\left(H_{2}^{\Delta}(t, s) A^{\sigma}(s)+H(t, s) A^{\Delta}(s)\right)^{2}}{4 \gamma H(t, s) A(s) \alpha(s)} .
\end{aligned}
$$

When $\gamma \geq 1$, we have

$$
\begin{aligned}
& H_{2}^{\Delta}(t, s) A^{\sigma}(s)\left(\frac{u(s)-B(s)}{A(s)}\right)^{\sigma}+H(t, s) \Phi_{0}(s) \\
& =\left(H_{2}^{\Delta}(t, s) A^{\sigma}(s)+H(t, s) A^{\Delta}(s)\right)\left(\frac{u(s)-B(s)}{A(s)}\right)^{\sigma} \\
& \quad-\gamma H(t, s) A(s)\left(\frac{\delta(s)}{r_{2}(s)}\right)^{1 / \gamma 2}\left[\left(\frac{u(s)-B(s)}{A(s)}\right)^{\sigma}\right]^{(1+\gamma) / \gamma} .
\end{aligned}
$$

Using the inequality

$$
\lambda a b^{\lambda-1}-a^{\lambda} \leq(\lambda-1) b^{\lambda},
$$

let $\lambda=\frac{1+\gamma}{\gamma}$, and

$$
\begin{aligned}
& a^{\lambda}=a^{(1+\gamma) / \gamma}=\gamma H(t, s) A(s)\left(\frac{\delta(s)}{r_{2}(s)}\right)^{1 / \gamma_{2}}\left[\left(\frac{u(s)-B(s)}{A(s)}\right)^{\sigma}\right]^{(1+\gamma) / \gamma}, \\
& b^{\lambda-1}=b^{1 / \gamma}=\frac{\gamma}{1+\gamma}\left(\frac{r_{2}(s)}{\delta(s)}\right)^{\gamma_{1} /(1+\gamma)} \frac{H_{2}^{\Delta}(t, s) A^{\sigma}(s)+H(t, s) A^{\Delta}(s)}{(\gamma H(t, s) A(s))^{\gamma /(1+\gamma)}},
\end{aligned}
$$

then we have

$$
\begin{aligned}
& H_{2}^{\Delta}(t, s) A^{\sigma}(s)\left(\frac{u(s)-B(s)}{A(s)}\right)^{\sigma}+H(t, s) \Phi_{0}(s) \\
& \quad \leq\left(\frac{r_{2}(s)}{\delta(s)}\right)^{\gamma_{1}} \frac{1}{(H(t, s) A(s))^{\gamma}}\left(\frac{H_{2}^{\Delta}(t, s) A^{\sigma}(s)+H(t, s) A^{\Delta}(s)}{1+\gamma}\right)^{1+\gamma} .
\end{aligned}
$$

Therefore, for all $\gamma>0$, by (18) we have

$$
\begin{aligned}
& \int_{t_{1}}^{t} H(t, s)\left(A(s) q(s)-B^{\Delta}(s)\right) \Delta s \\
& \quad \leq H\left(t, t_{1}\right) u\left(t_{1}\right)+\int_{t_{1}}^{t} H_{2}^{\Delta}(t, s) B^{\sigma}(s) \Delta s+\int_{t_{1}}^{t} \Phi_{1}(s) \Delta s
\end{aligned}
$$

which implies that

$$
\int_{t_{1}}^{t}\left[H(t, s)\left(A(s) q(s)-B^{\Delta}(s)\right)-H_{2}^{\Delta}(t, s) B^{\sigma}(s)-\Phi_{1}(s)\right] \Delta s \leq H\left(t, t_{1}\right) u\left(t_{1}\right) .
$$

Hence,

$$
\frac{1}{H\left(t, t_{1}\right)} \int_{t_{1}}^{t}\left[H(t, s)\left(A(s) q(s)-B^{\Delta}(s)\right)-H_{2}^{\Delta}(t, s) B^{\sigma}(s)-\Phi_{1}(s)\right] \Delta s \leq u\left(t_{1}\right)<\infty,
$$


which contradicts (17). So $z^{\Delta}(t)<0, t \in\left[t_{1}, \infty\right)_{\mathbb{T}}$, and it is clear that $\lim _{t \rightarrow \infty} z(t)$ exists. By Lemma 2.1 we see that $\lim _{t \rightarrow \infty} x(t)$ exists. The proof is completed.

When $\gamma \geq 1$, if (9) holds, we have the following corollary on the basis of Lemma 2.5 and Theorem 3.1.

Corollary 3.2 When $\gamma \geq 1$, assume that (9) holds and there exist $(A, B) \in(\mathscr{A}, \mathscr{B})$ and $H \in \mathscr{H}$ such that, for any $t_{1} \in\left[t_{0}, \infty\right)_{\mathbb{T}}$,

$$
\limsup _{t \rightarrow \infty} \frac{1}{H\left(t, t_{1}\right)} \int_{t_{1}}^{t}\left[H(t, s)\left(A(s) q(s)-B^{\Delta}(s)\right)-H_{2}^{\Delta}(t, s) B^{\sigma}(s)-\Phi_{1}(s)\right] \Delta s=\infty
$$

where

$$
\Phi_{1}(s)=\left(\frac{r_{2}(s)}{\delta(s)}\right)^{\gamma_{1}} \frac{1}{(H(t, s) A(s))^{\gamma}}\left(\frac{H_{2}^{\Delta}(t, s) A^{\sigma}(s)+H(t, s) A^{\Delta}(s)}{1+\gamma}\right)^{1+\gamma} .
$$

Then (1) is oscillatory or $\lim _{t \rightarrow \infty} x(t)=0$.

Remark 3.3 In Corollary 3.2, letting $(A, B)=(1,0)$, we can simplify (19) as

$$
\limsup _{t \rightarrow \infty} \frac{1}{H\left(t, t_{1}\right)} \int_{t_{1}}^{t}\left[H(t, s) q(s)-\left(\frac{r_{2}(s)}{\delta(s)}\right)^{\gamma_{1}} \frac{1}{H^{\gamma}(t, s)}\left(\frac{H_{2}^{\Delta}(t, s)}{1+\gamma}\right)^{1+\gamma}\right] \Delta s=\infty
$$

When $B=0$, (12) is simplified as

$$
u(t)=A(t) \frac{r_{1}(t)\left(\left(r_{2}(t)\left(z^{\Delta}(t)\right)^{\gamma_{2}}\right)^{\Delta}\right)^{\gamma_{1}}}{z^{\gamma}(t)}, \quad t \in\left[t_{1}, \infty\right)_{\mathbb{T}}
$$

Now we have the following theorem.

Theorem 3.4 Assume that there exists $A \in C_{\mathrm{rd}}^{1}\left(D_{0},(0, \infty)\right)$ such that, for any $t_{1} \in\left[t_{0}, \infty\right)_{\mathbb{T}}$,

$$
\limsup _{t \rightarrow \infty} \int_{t_{1}}^{t}\left[A(s) q(s)-\Phi_{2}(s)\right] \Delta s=\infty
$$

where

$$
\Phi_{2}(s)= \begin{cases}\left(\frac{r_{2}(s)}{\delta(s)}\right)^{\gamma_{1}} \frac{\left(A^{\Delta}(s)\right)^{2}}{4 \gamma A(s) \alpha(s)}, & 0<\gamma<1, \\ \left(\frac{r_{2}(s)}{\delta(s)}\right)^{\gamma_{1}} \frac{1}{A^{\gamma}(s)}\left(\frac{A^{\Delta}(s)}{1+\gamma}\right)^{1+\gamma}, & \gamma \geq 1 .\end{cases}
$$

Then (1) is oscillatory or $\lim _{t \rightarrow \infty} x(t)$ exists.

Proof Assume that (1) is not oscillatory. Without loss of generality, we may suppose that $x(t)$ is an eventually positive solution of (1). Similarly as in the proof of Theorem 3.1, we have $z(t)$ is eventually positive or $\lim _{t \rightarrow \infty} x(t)=0$.

If $\lim _{t \rightarrow \infty} x(t)=0$, the theorem is proved. If $z(t)$ is eventually positive, there exists $t_{1} \in\left[t_{0}, \infty\right)_{\mathbb{T}}$ such that $z(t)>0$, and either $z^{\Delta}(t)>0$ or $z^{\Delta}(t)<0$ holds for $t \in\left[t_{1}, \infty\right)_{\mathbb{T}}$ 
by Lemma 2.2. Assume that $z^{\Delta}(t)>0, t \in\left[t_{1}, \infty\right)_{\mathbb{T}}$. Let $u(t)$ be defined by (20). Then by Lemma 2.6, we have

$$
u^{\Delta}(t)+A(t) q(t)-\Phi_{0}(t) \leq 0
$$

where $\Phi_{0}(t)$ is simplified as

$$
\Phi_{0}(t)= \begin{cases}A^{\Delta}(t)\left(\frac{u(t)}{A A(t)}\right)^{\sigma}-\gamma A(t) \alpha(t)\left(\frac{\delta(t)}{r^{2}(t)}\right)^{\gamma_{1}}\left[\left(\frac{u(t)}{A(t)}\right)^{\sigma}\right]^{2}, & 0<\gamma<1, \\ A^{\Delta}(t)\left(\frac{u(t)}{A(t)}\right)^{\sigma}-\gamma A(t)\left(\frac{\delta(t)}{r_{2}(t)}\right)^{1 / \gamma_{2}}\left[\left(\frac{u(t)}{A(t)}\right)^{\sigma}\right]^{(1+\gamma) / \gamma}, & \gamma \geq 1 .\end{cases}
$$

When $0<\gamma<1$, we have

$$
\begin{aligned}
u^{\Delta}(t) \leq & -A(t) q(t)+A^{\Delta}(t)\left(\frac{u(t)}{A(t)}\right)^{\sigma}-\gamma A(t) \alpha(t)\left(\frac{\delta(t)}{r_{2}(t)}\right)^{\gamma_{1}}\left[\left(\frac{u(t)}{A(t)}\right)^{\sigma}\right]^{2} \\
= & -A(t) q(t)+\left(\frac{r_{2}(t)}{\delta(t)}\right)^{\gamma_{1}} \frac{\left(A^{\Delta}(t)\right)^{2}}{4 \gamma A(t) \alpha(t)} \\
& -\gamma A(t) \alpha(t)\left(\frac{\delta(t)}{r_{2}(t)}\right)^{\gamma_{1}}\left[\left(\frac{u(t)}{A(t)}\right)^{\sigma}-\left(\frac{r_{2}(t)}{\delta(t)}\right)^{\gamma_{1}} \frac{A^{\Delta}(t)}{2 \gamma A(t) \alpha(t)}\right]^{2} \\
\leq & -A(t) q(t)+\left(\frac{r_{2}(t)}{\delta(t)}\right)^{\gamma_{1}} \frac{\left(A^{\Delta}(t)\right)^{2}}{4 \gamma A(t) \alpha(t)} .
\end{aligned}
$$

When $\gamma \geq 1$, we have

$$
u^{\Delta}(t) \leq-A(t) q(t)+A^{\Delta}(t)\left(\frac{u(t)}{A(t)}\right)^{\sigma}-\gamma A(t)\left(\frac{\delta(t)}{r_{2}(t)}\right)^{1 / \gamma_{2}}\left[\left(\frac{u(t)}{A(t)}\right)^{\sigma}\right]^{(1+\gamma) / \gamma} .
$$

Using the inequality

$$
\lambda a b^{\lambda-1}-a^{\lambda} \leq(\lambda-1) b^{\lambda}
$$

let $\lambda=\frac{1+\gamma}{\gamma}$, and

$$
\begin{aligned}
& a^{\lambda}=a^{(1+\gamma) / \gamma}=\gamma A(t)\left(\frac{\delta(t)}{r_{2}(t)}\right)^{1 / \gamma_{2}}\left[\left(\frac{u(t)}{A(t)}\right)^{\sigma}\right]^{(1+\gamma) / \gamma}, \\
& b^{\lambda-1}=b^{1 / \gamma}=\frac{\gamma}{1+\gamma}\left(\frac{r_{2}(t)}{\delta(t)}\right)^{\gamma_{1} /(1+\gamma)} \frac{A^{\Delta}(t)}{(\gamma A(t))^{\gamma /(1+\gamma)}},
\end{aligned}
$$

then we have

$$
u^{\Delta}(t) \leq-A(t) q(t)+\left(\frac{r_{2}(t)}{\delta(t)}\right)^{\gamma_{1}} \frac{1}{A^{\gamma}(t)}\left(\frac{A^{\Delta}(t)}{1+\gamma}\right)^{1+\gamma} .
$$

Therefore, for all $\gamma>0$, we always have

$$
u^{\Delta}(t) \leq-A(t) q(t)+\Phi_{2}(t)
$$

which implies that

$$
A(t) q(t)-\Phi_{2}(t) \leq-u^{\Delta}(t) .
$$


Letting $t$ be replaced by $s$, and integrating (22) with respect to $s$ from $t_{1}$ to $t \in\left[\sigma\left(t_{1}\right), \infty\right)_{\mathbb{T}}$, we obtain

$$
\int_{t_{1}}^{t}\left[A(s) q(s)-\Phi_{2}(s)\right] \Delta s \leq-\int_{t_{1}}^{t} u^{\Delta}(s) \Delta s=u\left(t_{1}\right)-u(t)<u\left(t_{1}\right)<\infty
$$

which is a contradiction of $(21)$. So $z^{\Delta}(t)<0, t \in\left[t_{1}, \infty\right)_{\mathbb{T}}$, and as before, $\lim _{t \rightarrow \infty} z(t)$ and $\lim _{t \rightarrow \infty} x(t)$ exist. The proof is completed.

When $\gamma \geq 1$, if (9) holds, from Lemma 2.5 and Theorem 3.4 we have the following result.

Corollary 3.5 When $\gamma \geq 1$, assume that (9) holds and there exists $A \in C_{\mathrm{rd}}^{1}\left(D_{0},(0, \infty)\right)$ such that, for any $t_{1} \in\left[t_{0}, \infty\right)_{\mathbb{T}}$,

$$
\limsup _{t \rightarrow \infty} \int_{t_{1}}^{t}\left[A(s) q(s)-\left(\frac{r_{2}(s)}{\delta(s)}\right)^{\gamma_{1}} \frac{1}{A^{\gamma}(s)}\left(\frac{A^{\Delta}(s)}{1+\gamma}\right)^{1+\gamma}\right] \Delta s=\infty .
$$

Then (1) is oscillatory or $\lim _{t \rightarrow \infty} x(t)=0$.

Remark 3.6 It is not difficult to satisfy the conditions in Corollary 3.5. Indeed, letting $A=1$, by (9) we have (23). The condition (23) can be deleted in Corollary 3.5. Therefore, when $\gamma \geq 1$, assume that (9) holds, then it follows that (1) is oscillatory or $\lim _{t \rightarrow \infty} x(t)=0$.

Remark 3.7 Take $r_{1}(t)=1 / a_{2}(t), r_{2}(t)=1 / a_{1}(t), \gamma_{1}=\alpha_{2}, \gamma_{2}=\alpha_{1}, \gamma=1, p(t)=0, h(t)=t$, and $f(t, x)=q(t) f_{0}(x)$, where $f_{0}$ is equivalent to $f$ in Yu and Wang [15]. It is obvious that the conclusions in this paper extend the ones in [15]. Meanwhile, the proofs and results above may provide some enlightenment to the study of oscillation of higher-order nonlinear dynamic equations with nonpositive neutral coefficients on time scales.

\section{Examples}

In this section, the application of our oscillation criteria will be shown in two examples. Now we give the first example to demonstrate Theorem 3.1 (or Corollary 3.2).

Example 4.1 Let $\mathbb{T}=\bigcup_{n=1}^{\infty}[2 n-1,2 n]$. Consider the equation

$$
\left(t\left(\left(\frac{1}{t}\left(\left(x(t)-\frac{t-1}{2 t} x(t-2)\right)^{\Delta}\right)^{1 / 3}\right)^{\Delta}\right)^{5}\right)^{\Delta}+\frac{2+\sin t}{t} x^{5 / 3}(h(t))=0,
$$

where $r_{1}(t)=t, r_{2}(t)=1 / t, p(t)=(t-1) / 2 t, g(t)=t-2, \gamma_{1}=5, \gamma_{2}=1 / 3, \gamma=5 / 3, h(t) \geq t$, and $t_{0}=1$. By (C3) we have $p_{0}=1 / 2$, and by (C6) we take $q(t)=1 / t$. Since

$$
\int_{t_{0}}^{\infty} \frac{1}{r_{1}^{1 / \gamma_{1}}(t)} \Delta t=\int_{1}^{\infty} \frac{1}{t^{1 / 5}} \Delta t=\infty, \quad \int_{t_{0}}^{\infty} \frac{1}{r_{2}^{1 / \gamma_{2}}(t)} \Delta t=\int_{1}^{\infty} t^{3} \Delta t=\infty
$$

and

$$
\int_{t_{0}}^{\infty} q(t) \Delta t=\int_{1}^{\infty} \frac{1}{t} \Delta t=\infty,
$$


it is obvious that the coefficients of (24) satisfy (C1)-(C6) and (9). Letting $H(t, s)=(t-s)^{2}$ and $(A, B)=(s, 0)$, we have

$$
\delta(t)=\int_{t_{1}}^{t} \frac{\Delta s}{r_{1}^{1 / \gamma_{1}}(s)}=\int_{t_{1}}^{t} \frac{\Delta s}{s^{1 / 5}}=O\left(t^{4 / 5}\right)
$$

and

$$
\begin{aligned}
\Phi_{1}(s) & =\left(\frac{r_{2}(s)}{\delta(s)}\right)^{\gamma_{1}} \frac{1}{(H(t, s) A(s))^{\gamma}}\left(\frac{H_{2}^{\Delta}(t, s) A^{\sigma}(s)+H(t, s) A^{\Delta}(s)}{1+\gamma}\right)^{1+\gamma} \\
& =\left(\frac{s^{-1}}{O\left(s^{4 / 5}\right)}\right)^{5} \frac{1}{\left((t-s)^{2} s\right)^{5 / 3}}\left(\frac{O(s) \cdot O(s)+(t-s)^{2}}{8 / 3}\right)^{8 / 3}=O\left(s^{-26 / 3}\right) .
\end{aligned}
$$

Hence,

$$
\begin{aligned}
& \limsup _{t \rightarrow \infty} \frac{1}{H\left(t, t_{1}\right)} \int_{t_{1}}^{t}\left[H(t, s)\left(A(s) q(s)-B^{\Delta}(s)\right)-H_{2}^{\Delta}(t, s) B^{\sigma}(s)-\Phi_{1}(s)\right] \Delta s \\
& \quad=\limsup _{t \rightarrow \infty} \frac{1}{\left(t-t_{1}\right)^{2}} \int_{t_{1}}^{t}\left[(t-s)^{2}-O\left(s^{-26 / 3}\right)\right] \Delta s=\infty .
\end{aligned}
$$

That is, (19) holds. By Theorem 3.1 (or Corollary 3.2) we see that (24) is oscillatory or $\lim _{t \rightarrow \infty} x(t)=0$.

The second example illustrates Theorem 3.4.

Example 4.2 Let $\mathbb{T}=\bigcup_{n=0}^{\infty}\left[3^{n}, 2 \cdot 3^{n}\right]$. Consider the equation

$$
\left(\frac{1}{t^{2}}\left(\left(\sqrt{t}\left(\left(x(t)-\frac{1}{t} x\left(\frac{t}{3}\right)\right)^{\Delta}\right)^{5 / 3}\right)^{\Delta}\right)^{1 / 5}\right)^{\Delta}+\frac{2+t^{2}}{t^{2}\left(1+t^{2}\right)} x^{1 / 3}(h(t))=0,
$$

where $r_{1}(t)=1 / t^{2}, r_{2}(t)=\sqrt{t}, p(t)=1 / t, g(t)=t / 3, \gamma_{1}=1 / 5, \gamma_{2}=5 / 3, \gamma=1 / 3, h(t) \geq \sigma(t)$, and $t_{0}=1$. By (C3) we have $p_{0}=0$, and by (C6) we take $q(t)=1 / t^{2}$. Since

$$
\int_{t_{0}}^{\infty} \frac{1}{r_{1}^{1 / \gamma_{1}}(t)} \Delta t=\int_{1}^{\infty} t^{10} \Delta t=\infty, \quad \int_{t_{0}}^{\infty} \frac{1}{r_{2}^{1 / \gamma_{2}}(t)} \Delta t=\int_{1}^{\infty} \frac{1}{t^{3 / 10}} \Delta t=\infty
$$

and

$$
\int_{t_{0}}^{\infty} q(t) \Delta t=\int_{1}^{\infty} \frac{1}{t^{2}} \Delta t<\infty
$$

it is obvious that the coefficients of (25) satisfy $(\mathrm{C} 1)-(\mathrm{C} 7)$. Then, letting $(A, B)=\left(s^{2}, 0\right)$, we obtain

$$
\begin{aligned}
\delta(t) & =\int_{t_{1}}^{t} \frac{\Delta s}{r_{1}^{1 / \gamma_{1}}(s)}=\int_{t_{1}}^{t} s^{10} \Delta s=O\left(t^{11}\right) \\
\alpha(t) & =\left(\frac{\delta(t)}{r_{2}(t)}\right)^{(1-\gamma) / \gamma_{2}}\left(2 \int_{t}^{\infty} q(s) \Delta s\right)^{(1-\gamma) / \gamma} \\
& =\left(\frac{O\left(t^{11}\right)}{\sqrt{t}}\right)^{2 / 5}\left(O\left(t^{-1}\right)\right)^{2}=O\left(t^{11 / 5}\right),
\end{aligned}
$$


and

$$
\begin{aligned}
\Phi_{2}(s) & =\left(\frac{r_{2}(s)}{\delta(s)}\right)^{\gamma_{1}} \frac{\left(A^{\Delta}(s)\right)^{2}}{4 \gamma A(s) \alpha(s)} \\
& =\left(\frac{\sqrt{s}}{O\left(s^{11}\right)}\right)^{1 / 5} \frac{O\left(s^{2}\right)}{4 / 3 \cdot s^{2} \cdot O\left(s^{11 / 5}\right)}=O\left(s^{-43 / 10}\right) .
\end{aligned}
$$

Therefore,

$$
\limsup _{t \rightarrow \infty} \int_{t_{1}}^{t}\left[A(s) q(s)-\Phi_{2}(s)\right] \Delta s=\limsup _{t \rightarrow \infty} \int_{t_{1}}^{t}\left[1-O\left(s^{-43 / 10}\right)\right] \Delta s=\infty
$$

That is, (21) holds. By Theorem 3.4 we see that (25) is oscillatory or $\lim _{t \rightarrow \infty} x(t)$ exists.

\section{Competing interests}

The author declares that he has no competing interests.

\section{Acknowledgements}

This project was supported by the NNSF of China (no. 11271379).

Received: 18 May 2015 Accepted: 6 September 2015 Published online: 18 September 2015

\section{References}

1. Hilger, S: Ein Maßkettenkalkül mit Anwendung auf Zentrumsmannigfaltigkeiten. Ph.D. thesis, Universität Würzburg (1988)

2. Hilger, S: Analysis on measure chain - a unified approach to continuous and discrete calculus. Results Math. 18, 18-56 (1990)

3. Agarwal, RP, Bohner, M: Basic calculus on time scales and some of its applications. Results Math. 35, 3-22 (1999)

4. Agarwal, RP, Bohner, M, O'Regan, D, Peterson, A: Dynamic equations on time scales: a survey. J. Comput. Appl. Math. $141,1-26(2002)$

5. Bohner, M, Peterson, A: Dynamic Equations on Time Scales: An Introduction with Applications. Birkhäuser, Boston (2001)

6. Bohner, M, Peterson, A (eds.): Advances in Dynamic Equations on Time Scales. Birkhäuser, Boston (2003)

7. Erbe, L, Hassan, TS, Peterson, A: Oscillation of third order nonlinear functional dynamic equations on time scales. Differ. Equ. Dyn. Syst. 18(1), 199-227 (2010)

8. Hassan, TS: Oscillation of third order nonlinear delay dynamic equations on time scales. Math. Comput. Model. 49 1573-1586 (2009)

9. Li, Q, Wang, R, Chen, F, Li, TX: Oscillation of second-order nonlinear delay differential equations with nonpositive neutral coefficients. Adv. Differ. Equ. 2015, Article ID 35 (2015)

10. Qiu, YC, Wang, QR: Kamenev-type oscillation criteria of second-order nonlinear dynamic equations on time scales. Discrete Dyn. Nat. Soc. 2013, Article ID 315158 (2013). doi:10.1155/2013/315158

11. Qiu, YC, Wang, QR: Oscillation criteria of second-order dynamic equations with damping on time scales. Abstr. Appl. Anal. 2014, Article ID 964239 (2014). doi:10.1155/2014/964239

12. Qiu, YC: Nonoscillatory solutions to third-order neutral dynamic equations on time scales. Adv. Differ. Equ. 2014 Article ID 309 (2014)

13. Saker, SH, Agarwal, RP, O'Regan, D: Oscillation of second-order damped dynamic equations on time scales. J. Math Anal. Appl. 330(2), 1317-1337 (2007)

14. Senel, MT: Kamenev-type oscillation criteria for the second-order nonlinear dynamic equations with damping on time scales. Abstr. Appl. Anal. 2012, Article ID 253107 (2012). doi:10.1155/2012/253107

15. Yu, ZH, Wang, QR: Asymptotic behavior of solutions of third-order nonlinear dynamic equations on time scales. J. Comput. Appl. Math. 225, 531-540 (2009)

16. Zhang, SY, Wang, QR: Oscillation for third-order nonlinear dynamic equations on time scales. Acta Sci. Natur. Univ. Sunyatseni 51(4), 50-55 (2012) (in Chinese) 